\title{
Construyendo la integralidad a través de articulación universidad sociedad
}

\section{Building Integrality through the Integration between the University and the Society}

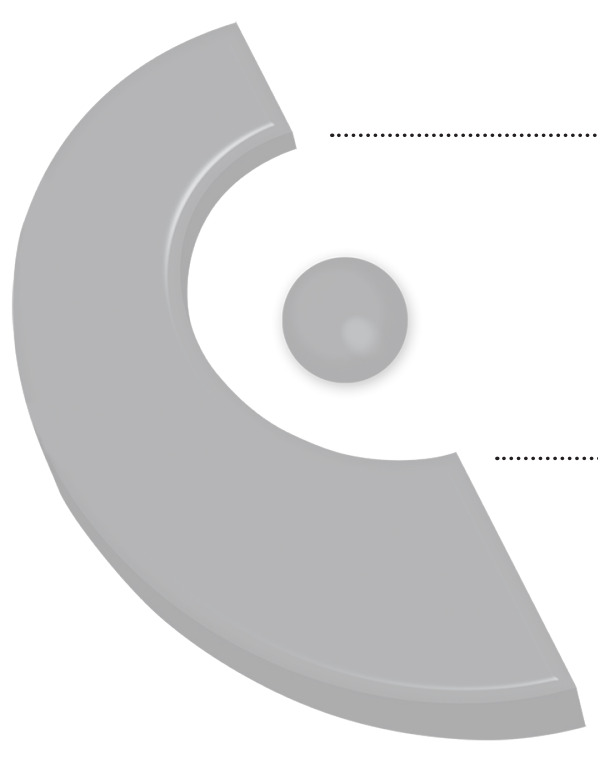

Giselle Hidalgo-Redondo

Universidad Nacional

Sede Regional Brunca, Coto guiselle.hidalgo.redondo@una.ac.cr

Recibido: 03/ 10 /2014 Aceptado: 28/06/2016

Resumen: Desarrollar proyectos que promueven la integralidad de las tres funciones esenciales de la universidad se problematiza en la Sede Regional Brunca Campus Coto en la Región Pacífico Sur, ya que no se cuenta con lineamientos que definan con claridad ejes temáticos que reflejen la interrelación con la comunidad. En la búsqueda de una solución que responda a esta problemática se propone a través de una metodología participativa implementar "Diálogos Comunitarios: vinculación Universidad-Comunidad" con el objetivo de abrir espacios de reflexión y discusión con el fin de generar información pertinente que facilite desarrollar proyectos que integren la docencia, la investigación y la extensión y que respondan a las necesidades priorizadas por la comunidad en una construcción colectiva, participativa y articulada del conocimiento académico-popular.

Palabras clave: integralidad, comunidad, universidad. 


\begin{abstract}
One problem at the Brunca Regional Campus of the Campus Coto in the South Pacific region is development of projects that promote the three essential functions of the university in a complete manner, since it does not have the guidelines to clearly define important subjects which reflect the interrelation with the community. In the quest for a solution to solve to this problem, a proposal is given, that employs a participative methodology, to implement "Community Dialogues: University-Community Links " with the objective of making available spaces of reflection and discussion in order to generate pertinent information that facilitates the development of projects that integrate teaching, research, and extension; and, that respond to the prioritized needs of the community in a collective, participative, and articulate construction of the academic-popular knowledge.
\end{abstract}

Keywords: completeness, community, university.

Todos sabemos algo y todos ignoramos algo, por eso aprendemos siempre.

Paulo Freire

\title{
Introducción
}

Podría conceptuarse enseñar y aprender, como una construcción de conocimiento académico-popular resultado de una simbiosis colectiva de las y los actores involucrados en determinado contexto, y momento histórico. Esto infiere que enseñar y aprender, aprender y enseñar como una reflexión profunda, respecto al objeto de conocimiento o problemática analizada.

La pregunta que se plantea es cómo académicos vinculamos la tríada: docencia, investigación y extensión con los y las estudiantes en los cursos de la carrera dentro de una comunidad, en donde la universidad como un actor más, debe aportar al bienestar social y a la libertad a través de proyectos que sean pertinentes que impacten el desarrollo social, económico y ambiental.

En cuanto a normativa el Estatuto Orgánico de la Universidad Nacional (1993) indica como la extensión, investigación y la docencia lo siguiente (ver tabla 1): 
Tabla 1

Normativa del Estatuto Orgánico de la Universidad Nacional (1993): extensión, investigación y docencia

\begin{tabular}{|c|c|c|}
\hline Extensión & Docencia & Investigación \\
\hline $\begin{array}{l}\text { "la tarea por medio de la cual } \\
\text { la Universidad se interrela- } \\
\text { ciona crítica y creadoramente } \\
\text { con la comunidad nacional. } \\
\text { Proyecta a la sociedad, de la } \\
\text { que la universidad forma par- } \\
\text { te, el producto de su quehacer } \\
\text { académico, a la vez que lo re- } \\
\text { dimensiona y enriquece al per- } \\
\text { cibir las auténticas y dinámicas } \\
\text { necesidades de la sociedad". }\end{array}$ & $\begin{array}{l}\text { "la acción planificada y sis- } \\
\text { temática de la enseñanza que } \\
\text { activa procesos de adquisición } \\
\text { y construcción de conocimien- } \\
\text { tos, actitudes y habilidades, a } \\
\text { partir de una relación abierta, } \\
\text { dentro de un currículum di- } \\
\text { námico y flexible. Es partici- } \\
\text { pativa, creativa, innovadora, } \\
\text { crítica y pluralista. La docen- } \\
\text { cia contribuye junto con la } \\
\text { investigación, la extensión y } \\
\text { la producción, al desarrollo in- } \\
\text { tegral del estudiante, así como } \\
\text { a la formación de los recursos } \\
\text { humanos que requiere la socie- } \\
\text { dad para su desarrollo". }\end{array}$ & $\begin{array}{l}\text { "la creación de conocimientos } \\
\text { es una función primordial de } \\
\text { la Universidad. Este proceso se } \\
\text { realiza por medio de sus diver- } \\
\text { sas actividades y áreas Acadé- } \\
\text { micas. La investigación es una } \\
\text { función fundamental del que- } \\
\text { hacer universitario. Debe ser } \\
\text { realizada por los miembros de } \\
\text { la comunidad institucional. Con } \\
\text { la investigación, la Universidad } \\
\text { genera y transfiere a la sociedad } \\
\text { el conocimiento y la tecnología } \\
\text { requeridos para satisfacer las } \\
\text { necesidades del país y coadyu- } \\
\text { var en su desarrollo humano, } \\
\text { económico y social. La inves- } \\
\text { tigación aporta a la extensión } \\
\text { y a la docencia, los elementos } \\
\text { básicos para el desempeño de } \\
\text { su quehacer y, a la vez, se retro- } \\
\text { alimenta de ambas". }\end{array}$ \\
\hline
\end{tabular}

Nota: elaboración propia en base a normativa del Estatuto Orgánico de la Universidad Nacional (1993)

La respuesta de cómo desarrollar proyectos que promuevan la integralidad de las tres funciones esenciales de la universidad será el resultado de aquellos(as) académicos(as) que visualicen los espacios vacíos, las ausencias y que lideren el quehacer universitario y motiven a otros a la excelencia, y como indicaba Leonardo Da Vinci: "hay tres clases de personas: aquellas que ven, aquellas que ven lo que se les muestra y aquellas que no ven".

Delimitando a la Sede Regional Brunca Campus Coto, esta se ubicada en la provincia de Puntarenas, cantón de Corredores en la Región Pacífico Sur; celebra este año 2014 su decimo aniversario y posee una población aproximada a los 400 estudiantes. 
Revista Universidad en Diálogo • Vol. 6, N. ํㄹㄹ Julio-diciembre 2016, pp. 241-252

ISSN 2215-2849 • EISSN: 2215-4752

DOI: http://dx.doi.org/10.15359/udre.6-2.13

En cuanto a proyectos de extensión e investigación se refiere en el Campus Coto los y las académicos afrontan incertidumbre en cuanto a ejes temáticos que sirvan de referente en el desarrollo de proyectos pertinentes que atiendan problemáticas priorizadas por la comunidad desde una perspectiva integral, en donde la desvinculación de la institución se hace evidente en ausencia de un proceso que manifieste el compromiso con sus aspiraciones y necesidades. Se requiere construir una respuesta en donde universidad y la comunidad se manifieste colectiva y participativamente.

En cuanto a la misión de la Sede Regional Brunca se indica a la letra: "Somos una sede de la universidad nacional, que desarrolla proyectos y programas de investigación, extensión, producción, y de formación de profesionales de excelencia con consciencia social y humanística, de acuerdo con las necesidades del desarrollo regional y nacional. Mediante una cultura de mejoramiento continuo de nuestro recurso humano, optimizando los recursos que la sociedad nos facilita e implementando las nuevas tecnologías de la comunicación e información, en beneficio de los sectores menos favorecidos de la Región Brunca dentro del contexto de la diversidad cultural, étnica y social que la caracteriza."

Dentro de su oferta académica al 2014 cuenta con cuatro bachilleratos: Gestión empresarial del Turismo Sostenible, Enseñanza del Inglés, Ingeniería en Sistemas de Información y Administración, además de dos licenciaturas: Sistemas de Información y Administración. Para el año 2015 se dejará de ofertar la carrera de Gestión empresarial del Turismo Sostenible.

La infraestructura instalada es de: seis aulas (dos aulas ocupadas por Colegio Humanístico Costarricense), un auditorio (capacidad aprox. 200 personas), una soda comedor, tres laboratorios (dos de computo y un laboratorio de idiomas, una planta de tratamiento de aguas residuales, amplias zona verde, pasillo espaciosos, residencia con capacidad para 24 estudiantes, plazoleta, una biblioteca, laboratorio portátil de computo (15 computadoras), internet inalámbrico. Se tiene proyectado para el año 2014-2015: construcción gimnasio (presupuesto piso y techo) y dos aulas.

Dentro de las instalaciones del Campus Coto el espacio para atender estudiantes por parte de los y las académicos(as) es muy reducido no mayor a los tres metros cuadrados, además y no se cuenta con área común docente que facilite labores administrativas para los proyectos, como fotocopiadora, teléfonos, fax, impresoras, entre otros. Inexistencia de comedor común administrativo y docente en donde almorzar o tomar café. El uso del transporte también es limitado en el Campus Coto, solamente se tiene un chofer, una microbús y un vehículo. 
La oferta docente es de 38 docentes, se detalla a continuación:

Tabla 2

Docentes, tiempo laborado y grado académico: Sede Regional Brunca Campus Coto

\begin{tabular}{ccccc}
\hline & \multicolumn{4}{c}{ Tiempo laborado para la institución } \\
\hline Docentes & $\begin{array}{c}\text { Tiempo } \\
\text { completo }\end{array}$ & $\begin{array}{c}\text { Medio } \\
\text { tiempo }\end{array}$ & $\begin{array}{c}\text { Cuarto } \\
\text { tiempo }\end{array}$ & Ocasionales \\
\hline 38 & 13 & 6 & 16 & 3 \\
\hline Grado académico & Doctorado & Maestría & Licenciatura & Bachiller \\
\cline { 2 - 5 } & 1 & 6 & 24 & 5 \\
\hline
\end{tabular}

Nota: elaboración propia en base a información brindada por la dirección del Campus Coto al segundo semestre 2013

Los proyectos inscritos con el Fondo Universitario para el Desarrollo Regional (Funder) y con contraparte de tres docentes del Campus Coto son los siguientes:

- Código 0119-12. Fortalecimiento de los sistemas productivos de las cooperativas de autogestión y otros grupos organizados de la zona sur de Costa Rica.

- Código: 0339-13. Construyendo espacios de diálogo intercultural desde el colegio humanístico en torno a los derechos de los pueblos indígenas

- Código: 0730-13. Germinadora de empresas, empleos y proyectos

El Fondo Universitario para el Desarrollo Regional (FUNDER) busca "estimular la formulación y ejecución de propuestas que promuevan el desarrollo regional e incentivar acciones académicas entre las sedes y otros programas regionales con las Facultades, Centros y Unidades Académicas de la Institución, incluidas las estaciones biológicas ubicadas en diversas regiones del país" (UNA Gaceta No 1, 28 de febrero del 2009).

\section{Diálogos Comunitarios: vinculación Universidad-Comunidad}

Se hace pertinente interiorizar y desconstruir conceptos como: extensión, investigación, docencia, educación y aprendizaje; como un proceso dialógico que contribuya a sensibilizar al docente en un proceso conjunto 
Revista Universidad en Diálogo • Vol. 6, N. 2, Julio-diciembre 2016, pp. 241-252

ISSN 2215-2849 • EISSN: 2215-4752

DOI: http://dx.doi.org/10.15359/udre.6-2.13

universidad-comunidad y conocimiento académico-popular, en enfatizar su papel como actor del cambio social y, como su actuar materializa esa vinculación ligado a los y las estudiantes como actores también desde la universidad inmersa en comunidad.

Este actuar se puede enunciar con la frase "Los profesores enseñan tanto por lo que saben como por lo que son". (Zabalza, 2002), libertando la sensibilización como parte del proceso de formación humanista en la cultura organizacional universitaria.

El diálogo entre ambas partes universidad-comunidad es el punto de partida, indicaba Paulo Freire (1970):

El diálogo es este encuentro de los hombres, mediatizados por el mundo, para pronunciarlo no agotándose, por lo tanto, en la mera relación yotú. Esta razón que hace imposible el diálogo entre aquellas que quieren pronunciar el mundo y los que no quieren, entre los que niegan a los demás el derecho a decir la palabra y aquellos a quienes se ha negado este derecho. Primero, es necesario que los que así se encuentran, negados del derecho primordial de decir la palabra, reconquisten ese derecho prohibiendo que continúe este asalto deshumanizante. (p.71)

Nos enfrentamos a tiempos de movimientos y cambios sociales, económicos y ambientales, ante un nuevo paradigma de mundo globalizado, y es importante resaltar lo expresado por Boaventura de Souza Santos (2006):

La extensión tendrá un significado muy especial en un futuro próximo. Precisamente cuando el capitalismo global pretende funcionalizar la universidad $\mathrm{y}$, de hecho, transformarla en una vasta agencia de extensión a su servicio, la reforma universitaria debe conceder una nueva importancia a las actividades de extensión (con implicaciones en el currículum y en las carreras de los docentes) y concebirlas de modo alternativo al capitalismo global, atribuyendo a las universidades una participación activa en la construcción de la cohesión social, en la profundización de la democracia, en la lucha contra la exclusión social y la degradación ambiental, en la defensa de la diversidad cultural” (p. 64).

En la búsqueda de una solución que responda a esta problemática se propone a través de una metodología participativa implementar Diálogos Comunitarios: vinculación Universidad-Comunidad con el objetivo de abrir espacios de reflexión y discusión con el fin de generar información pertinente que facilite desarrollar proyectos que integren la docencia, la inves- 
tigación y la extensión y que respondan a las necesidades priorizadas por la comunidad en una construcción colectiva, participativa y articulada del conocimiento académico-popular.

Se propone realizar al menos 100 diálogos en el cantón de Corredores, un diálogo por cada comunidad que se encuentre en los cuatro distritos: Corredor, la Cuesta, Canoas y Laurel. Se detalla a continuación:

Tabla 3

Diálogos Comunitarios: vinculación Universidad-Comunidad

\begin{tabular}{ll}
\hline $\begin{array}{c}\text { Diálogos Comunitarios: } \\
\text { vinculación Universidad-Comunidad }\end{array}$ \\
\hline Cantón de Corredores & Distrito Corredores: Neily. Ciudad. \\
& Barrios: Bosque, Caño Seco, Capri, Carmen, Corredor, \\
& Progreso, San Juan, Valle del Sur. \\
& Poblados: Abrojo, Aguilares, Alto Limoncito, Bajo \\
& Indios, Betel, Cacoragua, Campiña,Campo Dos, Campo \\
& Dos y Medio, Cañada, Caracol Sur, Castaños, Coloradito, \\
& Concordia, Coto 42, Coto 44, Coto 45, Coto 47, Coto 49, \\
& Coto 50-51, Coto 52-53, Cuesta Fila de Cal, Estrella del \\
& Sur, Florida, Fortuna, Kilómetro 10, Miramar, Montezuma, \\
& Nubes, Pangas, Planes, Pueblo Nuevo, Río Bonito, Río \\
& Nuevo (Norte), Río Nuevo (Sur), San Antonio Abajo, San \\
& Francisco, San Josecito, San Rafael, Santa Cecilia, Santa \\
& Marta (parte), Santa Rita, Tropezón, Unión, Vegas de \\
& Abrojo, Villa Roma.
\end{tabular}

Distrito La Cuesta: Villa.

Poblados: Canoas Abajo (parte), Control, Cuervito, Chorro.

Distrito Canoas: Villa.

Barrio: Lotes (San Jorge).

Poblados: Altos del Brujo, Bajo Brujo, Bajo, Barrionuevo, Canoas Abajo (parte), Canoas Arriba, Cañaza, Cerro Brujo, Colorado, Chiva, Darizara, Gloria, Guay, Guayabal, Mariposa, Níspero, Palma, Paso Canoas, San Antonio, San Isidro, San Martín, San Miguel, Santa Marta (parte), Veguitas de Colorado, Veracruz, Villas de Darizara.

continúa 


\begin{tabular}{l} 
Diálogos Comunitarios: \\
vinculación Universidad-Comunidad \\
\hline Distrito Laurel: Villa \\
Poblados: Alto Vaquita, Bambito, Bella Luz, Bijagual, \\
Caimito, Cangrejo Verde, Caracol de la Vaca, Cariari, \\
Caucho, Cenizo, Colonia Libertad, Coyoche, Jobo Civil, \\
Kilómetro 22, Kilómetro 25, Kilómetro 27, Kilómetro \\
29, Mango, Pueblo de Dios, Puerto González Víquez, \\
Río Incendio, Roble, San Juan, Santa Lucía, Tamarindo, \\
Vaca (Santa Rosa), Vereh, Zaragoza. \\
Representantes de las organizaciones sociales, públicos \\
y privados. \\
\hline Primera etapa: actores involucrados. \\
- Mapeo de los actores de cada distrito por tipo de \\
organización. \\
Segunda etapa: Diálogo. \\
- Definir un espacio neutral dentro de la comunidad \\
en donde reunirse con las los actores de las \\
organizaciones sociales, públicas y privadas. Se espera \\
que cada diálogo tenga al menos 20 participantes. \\
- Dinámica de presentación: la telaraña. \\
- Iniciar con el significado de la palabra “dialogo" y en \\
el cómo establecemos un diálogo. \\
- Técnica de tarjetas activas: Pregunta generadora: ¿qué \\
problemas enfrenta mi comunidad? Cada persona en \\
el taller escribirá el problema identificado. Luego se \\
agruparan en la pared los problemas similares y se \\
priorizarán por los participantes. \\
- Mesas de trabajo: se dividirán los participantes en \\
grupos de no más de cinco miembros y se asignarán \\
aleatoriamente los cuatro problemas priorizados. \\
Arbol de problemas: identificación de causas y efectos, \\
hacia el árbol de objetivos. \\
\end{tabular}




\begin{tabular}{ll}
\hline & \multicolumn{1}{c}{ Diálogos Comunitarios: } \\
& vinculación Universidad-Comunidad \\
\hline & Tercera etapa: definición proyectos priorizados por la \\
& comunidad. \\
& Cuarta etapa: formulación, ejecución, y cierre del \\
& proyecto. \\
& Teniendo como línea base definida por los actores \\
& sociales, públicos y privados los proyectos definidos a \\
& implementar. \\
& Quinta etapa: accesibilidad a fondos económicos: \\
& FUNDER, Fondos del Sistema, CRUSA, entre otros. \\
\hline Información & Cada proyecto deberá sistematizar sus procesos y \\
generada de los & resultados y publicar al menos un artículo por año. \\
diálogos & Con una línea base definida de proyectos, se insta \\
\hline $\begin{array}{l}\text { Actividades } \\
\text { integrales por cada } \\
\text { curso de carrera }\end{array}$ & $\begin{array}{l}\text { a los académicos a involucrar a sus estudiantes en } \\
\text { gestores del cambio social en su quehacer como futuros } \\
\text { profesionales. }\end{array}$ \\
\hline
\end{tabular}

Nota: elaboración propia en base a experiencia en proyectos interuniversitarios de regionalización y los distritos en base a la Gaceta (2009, 26 de mayo). División Territorial Administrativa de Costa Rica. La Gaceta, p. 99-100.

Los Diálogos Comunitarios: vinculación Universidad-Comunidad, ofrecerán una línea base para la ejecución de proyectos, sin embargo es importante considerar también la estrategia general de desarrollo identificada en Plan de Desarrollo Humano Local del Cantón de Corredores 2013-2023, la cual se indica a la letra: "desarrollar un cantón inclusivo, equitativo y seguro por medio del trabajo institucional y participativo de sus comunidades en pro de garantizar mejor calidad de vida para sus habitantes". Aunado a las actividades integrales con los y las estudiantes de los cursos de las cuatro carreras; encuadrados en la integralidad la docencia, investigación y extensión y en un contexto universidad-comunidad. 

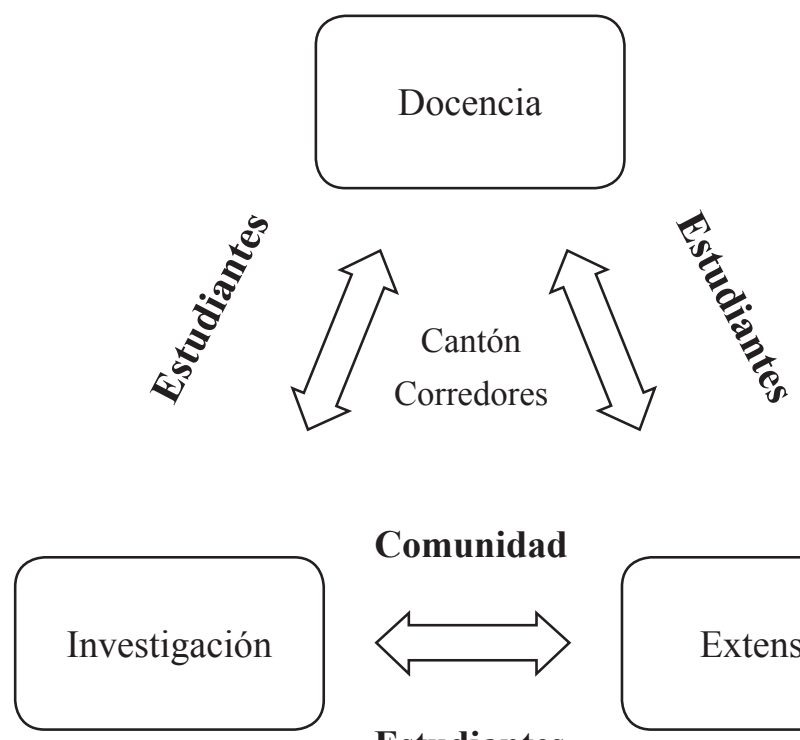

\section{Comunidad}

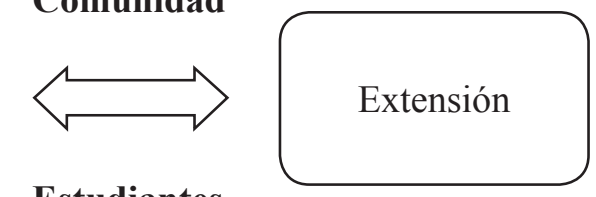

\section{Estudiantes}

Plan de Desarrollo Humano Local del Cantón de Corredores 2013-2023

Figura 1: Diálogos comunitarios: vinculación universidad-comunidad. Sede Regional Brunca Campus Coto.

Nota: Elaboración propia.

\section{Conclusiones}

....el país vive "una situación límite, un tiempo de inflexión en su desarrollo humano sostenible, marcado por el deterioro institucional y por la imposibilidad del sistema político de adaptarse y responder a la población con entregas efectivas de un mayor y más equitativo bienestar social, económico y ambiental. (XVII Informe del Estado de la Nación. San José, 2011)

Se requiere sensibilizar a las y los académicos para que promuevan actividades integrales con los y las estudiantes dentro de cada curso para que se proyecten dentro de la comunidad con una intencionalidad de convergencia transversal en la vinculación universidad comunidad.

La construcción de una respuesta que busque soluciones, requiere cuestionarnos en nuestro quehacer académico, y exhorta a ver más allá desde una perspectiva 
creadora, humanizada y sensibilizada, se insta a reflexionar sobre el concepto “académico(a)", y cuáles deberán ser sus valores y competencias. Además se debe considerar dentro de la acreditación curricular la implementación de requisitos que deban cumplirse que ayuden a sensibilizar su participación en proyectos con la comunidad. Asimismo, cada proyecto que se sistematice, deberá publicar un artículo mínimo por año, e instar al menos una actividad integral en cada curso de cada carrera, esto con el fin de vincular a los estudiantes con la problemática social y las organizaciones sociales, públicas y privadas.

Dentro del organigrama organizacional de la universidad se hace necesaria la figura de un coordinador(a) regional de enlace en cada sede que actúe en el acompañamiento de los procesos y procedimientos en las etapas de formulación, ejecución, seguimiento y cierre de los proyectos. Si en cada sede se instaurara esta figura de enlace, se facilitaría la comunicación del quehacer universitario desde la región y hacia la Sede Central Campus Omar Dengo con una red de enlace que englobe los proyectos de extensión e investigación, rescatando así, la generación de conocimiento a través de compartir experiencias y resultados ante un proceso articulado.

Además de dar seguimiento a proyectos gestados desde la Sede Central Campus Omar Dengo que se implementan en las regiones y que muchas veces actúan de manera paralela a las sedes regionales. Cito por ejemplo el caso de la Universidad de Costa Rica que dentro de la estructura organizacional tiene instaurada desde la Vicerrectoria de Acción Social, a la Comisión de Acción Social (CAS), la indica a la letra que" es una comisión ordinaria y permanente que se establece en cada unidad académica para optimizar el buen funcionamiento de las actividades de Acción Social mediante el análisis de las propuestas y los resultados que presenten los y las docente".

Como docentes debemos apuntar a la excelencia académica, a nuestra transformación y desarrollo profesional en forma permanente. "Los profesores enseñan tanto por lo que saben como por lo que son". Esta vieja sentencia pedagógica ha recibido escasa atención en el contexto universitario. Se diría que la dimensión personal del profesorado desaparece o se hace invisible en el ejercicio profesional. Lo que uno mismo es, siente o vive, las expectativas con las que desarrolla su trabajo se desconsideran como variables que pudieran afectar la calidad de la enseñanza. Pero parece claro que no es así y que buena parte de nuestra capacidad de influencia en los estudiantes se deriva precisamente de lo que somos como personas, de nuestra forma de presentarnos, de nuestras modalidades de relación con ellos (Zabalza, Miguel Angél, et al; 2002) 
Revista Universidad en Diálogo • Vol. 6, N. 2 2, Julio-diciembre 2016, pp. 241-252

ISSN 2215-2849 • EISSN: 2215-4752

DOI: http://dx.doi.org/10.15359/udre.6-2.13

Retomar el concepto de ecología de saberes como "un conjunto de prácticas que promueven una nueva convivencia activa de saberes con el supuesto de que todos ellos, incluido el saber cientifico, se pueden enriquecer en ese diálogo. Implica una amplia gama de acciones de valoración, tanto del conocimiento científico como de otros conocimientos prácticos considerados útiles, compartidos por investigadores, estudiantes y grupos de ciudadanos, sirve de base para la creación de comunidades epistémicas más amplias que convierten a la universidad en un espacio público de interconocimiento donde los ciudadanos y los grupos sociales pueden intervenir sin la posición exclusiva de aprendices" (Santos, 2006: 67-68).

Esta reflexión es un acercamiento en la búsqueda de una respuesta que colectivamente deberá gestarse, sin embargo se requiere del apoyo institucional para poder concretarse y el reconocer que las ausencias están presentes en la materialización del vínculo universidad comunidad y resaltar el concepto de la regionalización desde y para las sedes regionales, aunque se requiera tiempo en el desarrollo de competencias y capacidades.

\section{Referencias}

Freire, P. (1970). Pedagogía del Oprimido. Madrid: Siglo: XXI.

La Gaceta (26 de mayo de 2009). División Territorial Administrativa de Costa Rica. La Gaceta, pp. 99-100.

Programa de las Naciones Unidas para el Desarrollo y Ministerio de Planificación Nacional y Política Económica. (2012). Plan de Desarrollo Humano Local del Cantón de Corredores 2013-2023. Recuperado de: http://www.ifam.go.cr/corredores/Part303/Plan\%20 Cantonal\%20de\%20Desarrollo\%20Humano\%20Local\%20Corredores\%202013-2023.pdf

UNA Gaceta. (2009). Reglamento del Fondo Universitario de Estímulo para el Desarrollo Regional (FUNDER). 28 de febrero. N.1.

Santos, B.S de Souza, (2006) La Universidad en el siglo XXI. Para una reforma democrática y emancipadora de la universidad. La Habana: CASA, Fondo Editorial Casa de las Américas.

Universidad de Costa Rica. Comisiones de Acción Social. Recuperado de: http://accionsocial.ucr.ac.cr/comisiones-accion-social

Zabalza-Beraza, M.A. (2002): La enseñanza universitaria: el escenario y sus protagonistas. Madrid: Narcea Ediciones. 